\title{
Deficits in nonlinear EEG synchronisation during open heart surgery: A pilot exploratory work.
}

\section{Veliziotis'1, N. Sarrigiannis1, R. C. Laguna², Y. Zhao², Y. Kapessidou1', P. G. Sarrigiannis ${ }^{3}$}

Service d'Anesthésie-Réanimation C.H.U. Saint-Pierre - Bruxelles (Belgium),

2 Through-life Engineering Services, Cranfield University, (United Kingdom),

3 Royal Hallamshire Hospital - Sheffield (United Kingdom)

\section{Background and Goal of Study:}

Postoperative cognitive dysfunction has been reported in $50 \%$ of patients after cardiac surgery. Dysfunction in nonlinear interactions between brain network regions have been previously shown in patients with cognitive deficits. The aim of this study was to explore whether such changes in nonlinear interactions can be detected during cardiopulmonary bypass (CPB).

\section{Materials and Methods:}

After approval by local ethics comity \& informed consent, 5 similar patients (2M-3F,age:53-81,ASAIII-IV) were included. Anesthesia was provided using propofol-remifentanil in TCI mode and standard neuroprotection technics (hypothermia \& MAP> $>00 \mathrm{mmHg}$ ). We have analysed frontopolar homologous EEG recordings (using 10-20 system of electrode placement). We used the NeuroSENSE monitor, recording at $256 \mathrm{~Hz}$ with the WAVcns function to help maintain the depth of anesthesia throughout surgery at the recommended level (between 40-60). We have selected two one minute epochs of EEG data, one several minutes before cannulation and a second after beginning of CPB.

\section{Results and Discussion:}

The Morlet wavelets power spectrum and the magnitude of the wavelet cross spectra, between the homologous frontopolar recordings, show no significant differences on the results produced for the EEG epoch before and after CPB. However, 4 of 5 cases show a drop in the magnitude of the Cross-bispectrum (a technique assessing nonlinear interactions) that ranged between $54-93 \%(p<0.04)$ in the post CPB EEG epoch. The significant drop in the magnitude of the Cross-bispectrum revealed in the postCPB EEG epochs, in comparison to the pre-cannulation EEG findings, suggests a selective relative attenuation in the intensity of nonlinear brain interactions. These findings were not reflected in the wavelet spectral and crossspectral analysis, in keeping with a consistent depth of anesthesia throughout surgery.

No changes in the magnitude of the cross-bispectrum interactions were seen in one of the 5 cases, militating against the differences found in the remaining patients being a reflection of the cooling effect on brain function.

\section{Conclusion(s):}

We show evidence of dampening of nonlinear interactions between homologous frontopolar areas on EEG recordings during CPB. None of the remaining linear quantitative estimates show significant changes. This finding needs further confirmation on a larger cohort of patients while exploring if this technique correlates with negative cognitive outcomes.

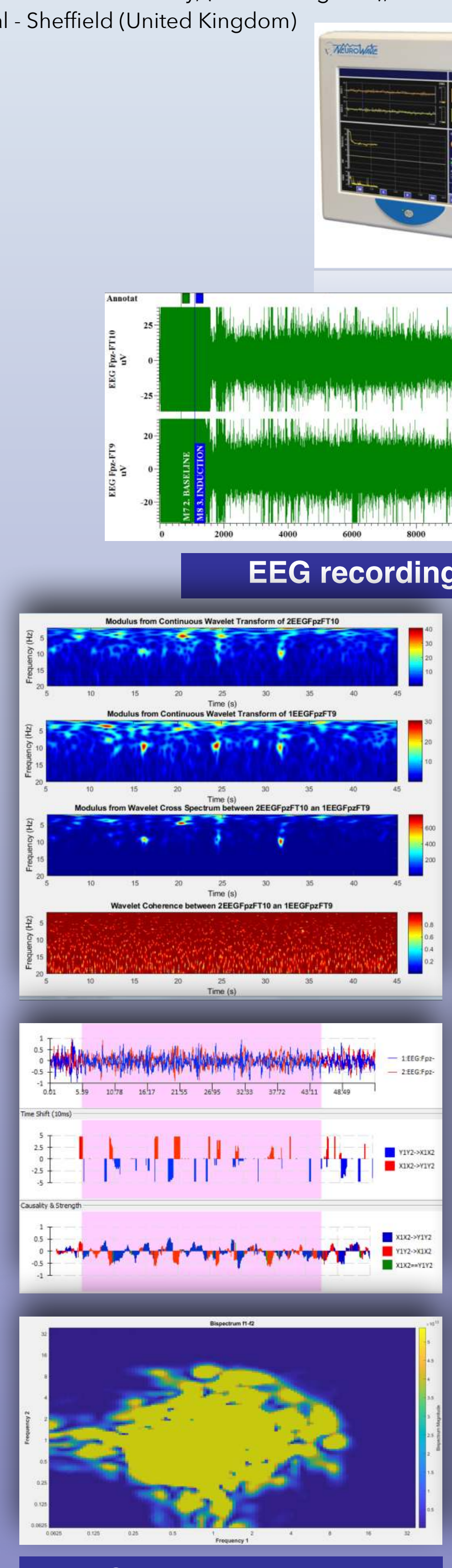

Steady state anesthesia 32 minutes prior to cannulation

\begin{tabular}{c|ccc|cccc}
\multicolumn{4}{c}{} & \multicolumn{3}{c|}{ Pre cannulation } & \multicolumn{3}{c}{ Post CPB } \\
\cline { 2 - 7 } ERR & Syn & Ave & Std & Syn & Ave & Std \\
\hline Case 1 & $5 \%$ & 0,22 & 0,16 & $3 \%$ & 0,21 & 0,15 \\
Case 2 & $7 \%$ & 0,24 & 0,16 & $4 \%$ & 0,15 & 0,11 \\
Case 3 & $16 \%$ & 0,25 & 0,13 & $15 \%$ & 0,26 & 0,14 \\
Case 4 & $35 \%$ & 0,4 & 0,21 & $31 \%$ & 0,35 & 0,19 \\
Case 5 & $32 \%$ & 0,39 & 0,23 & $18 \%$ & 0,38 & 0,21 \\
& & & & & & \\
Wavelet & Max Power R Cross BsP & Cross S & Max Power R Cross BsP & Cross S \\
\hline Case 1 & 30 & $5.3 \times 10^{13}$ & 800 & 30 & $5.5 \times 10^{12}$ & 1000 \\
Case 2 & 30 & $10 \times 10^{13}$ & 1800 & 30 & $8.5 \times 10^{12}$ & 800 \\
Case 3 & 15 & $3 \times 10^{12}$ & 300 & 15 & $14 \times 10^{11}$ & 300 \\
Case 4 & 30 & $2.5 \times 10^{16}$ & 1500 & 50 & $2.5 \times 10^{16}$ & 1500 \\
Case 5 & 40 & $9 \times 10^{16}$ & 1500 & 60 & $7 \times 10^{15}$ & 2500
\end{tabular}

